\title{
Observation of the Goos-Hänchen Shift with Neutrons
}

\author{
Victor-O. de Haan, Jeroen Plomp, Theo M. Rekveldt, Wicher H. Kraan, and Ad A. van Well \\ Department Radiation, Radionuclides \& Reactors, Faculty of Applied Sciences, Delft University of Technology, \\ Mekelweg 15, 2629 JB Delft, The Netherlands
}

Robert M. Dalgliesh and Sean Langridge

STFC, ISIS, Rutherford Appleton Laboratory, Chilton, Oxfordshire, OX11 OQX, United Kingdom (Received 27 August 2009; revised manuscript received 24 October 2009; published 8 January 2010)

\begin{abstract}
The Goos-Hänchen effect is a spatial shift along an interface resulting from an interference effect that occurs for total internal reflection. This phenomenon was suggested by Sir Isaac Newton, but it was not until 1947 that the effect was experimentally observed by Goos and Hänchen. We provide the first direct, absolute, experimental determination of the Goos-Hänchen shift for a particle experiencing a potential well as required by quantum mechanics: namely, wave-particle duality. Here, the particle is a spinpolarized neutron reflecting from a film of magnetized material. We detect the effect through a subtle change in polarization of the neutron. Here, we demonstrate, through experiment and theory, that neutrons do exhibit the Goos-Hänchen effect and postulate that the associated time shift should also be observable.
\end{abstract}

DOI: 10.1103/PhysRevLett.104.010401

The longitudinal Goos-Hänchen shift is well known for photons. It was Sir Isaac Newton [1] who suggested a beam of light, specularly reflected at a glass-vacuum surface, first penetrates into the vacuum and then is attracted back toward the glass surface. The result is a shift of the incident ray over some distance along the surface of the interface as shown in Fig. 1. F. Goos and H. Hänchen were the first to observe such a shift experimentally [2,3]. Since then, this shift for electromagnetic waves have been subject of further studies [4,5], including also transverse shifts, known as the Imbert-Fedorov shift [6]. Because of the particlewave duality, as advocated by quantum mechanics, such a shift should also be observable for particles reflecting from a potential well. As a neutron possesses a magnetic moment, the neutron wave function is represented by a spinor, consisting of an up-spin wave function and a down-spin wave function. The Goos-Hänchen shift for an up-spin wave function is different from the one for a down-spin wave function due to the difference in potential well for the spin up and down wave functions. The difference in these shifts results in a different polarization after reflection from the mirror with respect to the polarization before reflection.

For neutrons, experimental possibilities have been discussed $[7,8]$ previously, but to the best of the authors' knowledge, these experiments have not been performed. The measurements described by Pleshanov [9] are indirect measurements of the shift, determining the effect on the reflectivity, similar as the ones described by Toperverg [10]. Experimentally, to observe this change in polarization requires a high neutron flux instrument with excellent control and characterization of the neutron spin polarization. The development of the spin-echo neutron reflectometer OffSpec [11,12] at ISIS second target station enables measurement of the difference in Goos-Hänchen
PACS numbers: 03.75.Be, 03.65.Ta, 03.75.Dg, 42.50.Xa

shift via the direct and unambiguous measurement of the polarization change during reflection.

The practical importance of the shift for neutrons is for the design of neutron waveguides as discussed by Rohwedder [13] similar to Pillon [14] and it can also be used to study the coherence properties of a neutron beam, as will be discussed later.

For particle-wave functions, where interaction with magnetic and electric forces can not be ignored, Dirac [15] was able to reduce the time-dependent Schrödinger equation using two linear dependent solutions $\Psi^{+}(\vec{r}, t)$ and $\Psi^{-}(\vec{r}, t)$ representing the up-spin state and down-spin state part of the wave function. The relevant terms of the Hamiltonian are

$$
\hat{H}(\vec{r}, t)=-\frac{\hbar^{2}}{2 m} \nabla^{2}+V(\vec{r})-\mu_{n} \hat{\sigma} \cdot \vec{B}(\vec{r}, t),
$$

where $m$ is the neutron mass, $\hat{\sigma}$ the Pauli spin matrix vector, $V(\vec{r})$ is the complex nuclear optical potential, $\mu_{n}=$ $-60.308 \mathrm{neV} / \mathrm{T}$ the neutron magnetic moment, and $\vec{B}(\vec{r}, t)$ the magnetic flux density [16]. The quantity $\mu_{n} B(\vec{r}, t)$ is known as the Zeeman energy as it describes the energy gain or loss of a neutron experiencing a magnetic flux density. The Zeeman energy must be compared to the total kinetic energy of the neutron given by $\hbar^{2} k^{2} / 2 m$ ( $\approx 20.5 \mathrm{meV}$ for neutrons with a wavelength in vacuum, $\lambda$ of $0.2 \mathrm{~nm}$ ), where $k$ is the wave number of the neutron equal to $2 \pi / \lambda$. For moderate values of the magnetic flux density of about $0.3 \mathrm{~T}$, the Zeeman energy is 6 orders of magnitude smaller than the kinetic energy.

For reflection from a smooth interface, as shown in Fig. 1, the reflected wave function can be expressed in the incoming wave function and a reflection coefficient. If the scattering potential $V(\vec{r})$ and the magnetic flux density 


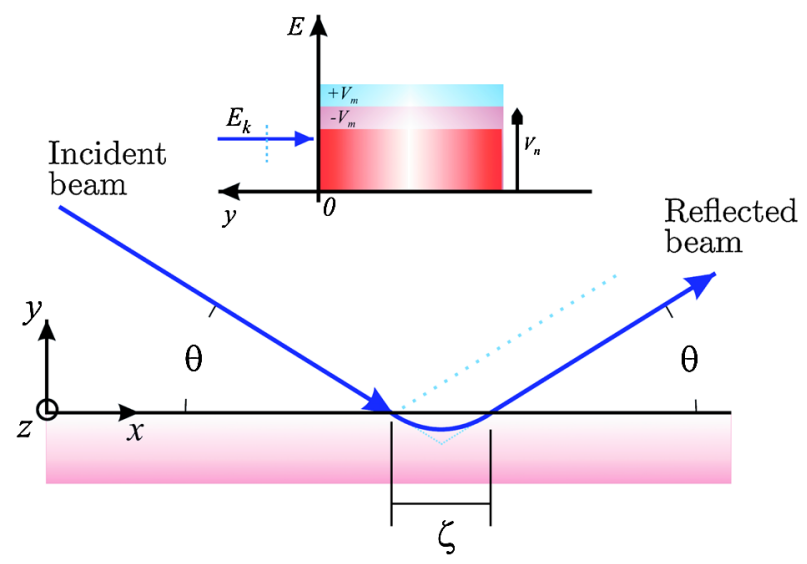

FIG. 1 (color online). Reflection of a plane wave with incident angle $\theta$ on a substrate boundary at $y=0$ indicating the GoosHänchen shift, $\zeta$. Inset: nuclear $V_{n}$ and magnetic $\pm V_{m}$, scattering potential and kinetic energy $E_{k}$ associated with neutron velocity component perpendicular to the surface.

$\vec{B}(\vec{r})$ are statistically stationary and only a function of the direction perpendicular to the sample surface (here the $y$ direction) the three-dimensional time-dependent Schrödinger equation can be reduced to a one-dimensional time-independent equation. Because the Hamiltonian only contains time-independent terms, the wave function can be split into a time-dependent and time-independent part. Further, the time-independent part reduces to two homogeneous second order differential equations for $x$ and $z$. For the general case, the solution for the remaining coupled nonhomogeneous differential equations for $y$ is discussed elsewhere (for instance, [17]). Here, the situation is discussed where the neutron reflects from the vacuumsubstrate interface and the magnetic flux density is parallel to the $z$ direction (hence, parallel to the interface and perpendicular to the neutron beam direction), where for $y \leq 0: V(y)=V_{n}$, the nuclear scattering potential of the substrate and $\vec{B}(y)$ is a vector in the $z$ direction with length $B_{s}$, the magnetic flux density inside the substrate, while for $y>0$ both quantities are 0 . The guide field outside the sample can be ignored. In this case, $\hat{\sigma} \cdot \vec{B}(y)=B(y) \hat{\sigma}_{z}$ and the two coupled differential equations can be reduced to two independent differential equations. The general solutions for these are for $y>0$,

$$
\psi_{k_{y}}^{ \pm}(y)=\alpha^{ \pm}\left(k_{y}\right)\left(e^{i k_{y} y}+\rho^{ \pm}\left(k_{y}\right) e^{-i k_{y} y}\right)
$$

where $k_{y}$ is the component of the wave vector in the $y$ direction, $\rho^{ \pm}\left(k_{y}\right)=\left(k_{y}-k_{y}^{s, \pm}\right) /\left(k_{y}+k_{y}^{s, \pm}\right)$ are the corresponding (Fresnel) reflection coefficients, where $k_{y}^{s, \pm}=$ $\sqrt{k_{y}^{2}-\left(k_{c}^{ \pm}\right)^{2}}$, is the wave vector component in the $y$ direction inside the substrate for up $(+)$ or down $(-)$ spin state. The corresponding critical wave vector is given by $k_{c}^{ \pm}=\sqrt{\frac{2 m}{\hbar^{2}}\left(V_{n} \mp V_{m}\right)}$, where $V_{m}=\mu_{n} B_{s}$ is the magnetic scattering potential. The first term of the right-hand side of Eq. (2) is interpreted as the incident neutron wave function and the second term as the reflected wave function. At the interface, the reflected wave function can be expressed in terms of the incident wave function according to $\psi_{k_{y}}^{ \pm}(0)_{\text {out }}=\rho^{ \pm}\left(k_{y}\right) \psi_{k_{y}}^{ \pm}(0)_{\text {in }}$. Here, the fact was used that no spin flip occurs at the interface as the magnetic flux density is parallel to the surface ( $z$ direction). Hence, the up-spin state and down-spin state are reflected independently according to the Fresnel reflection law. As long as the kinetic energy, $E_{k}=\hbar^{2} k_{y}^{2} / 2 m$ associated with the perpendicular component of the wave vector $k_{y}$ is larger than the scattering potential of the substrate, both $k_{y}$ and $k_{y}^{s, \pm}$ are real; hence, the reflection coefficient is real and the phase of the reflected beam is equal to the phase of the incident beam. However, as soon as this kinetic energy is smaller than the scattering potential, $k_{y}<k_{c}^{ \pm}, k_{y}^{s, \pm}$ becomes imaginary and total external reflection occurs. The amplitude of the reflection coefficient is 1 , but the phase of the reflected beam is shifted by $\phi^{ \pm}=-i \ln \left(\rho^{ \pm}\right)=2 \arccos \left(k_{y} / k_{c}^{ \pm}\right)$. This is the phase shift a plane wave experiences when reflecting from the interface. Such a phase shift can be translated into a spatial displacement for a beam of finite size. Following the reasoning of Renard [18], based on flux conservation for light or particles, the Goos-Hänchen shift can be calculated as

$$
\zeta^{ \pm}=\frac{k}{\left(k_{c}^{ \pm}\right)^{2}} \frac{2 k_{y}}{\sqrt{\left(k_{c}^{ \pm}\right)^{2}-k_{y}^{2}}} .
$$

This was later confirmed by theoretical calculations for a light beam of limited dimensions by Lotsch [19]. This shift as function of $k_{y}$ is shown in Fig. 2 for fully magnetized iron $\left(B_{s}=2.13 \mathrm{~T}\right.$, so $k_{c}^{+}=0.128 \mathrm{~nm}^{-1}$ and $k_{c}^{-}=$ $0.062 \mathrm{~nm}^{-1}$ ). Close to the critical edges, the shifts are of the order of tens of $\mu \mathrm{m}$.

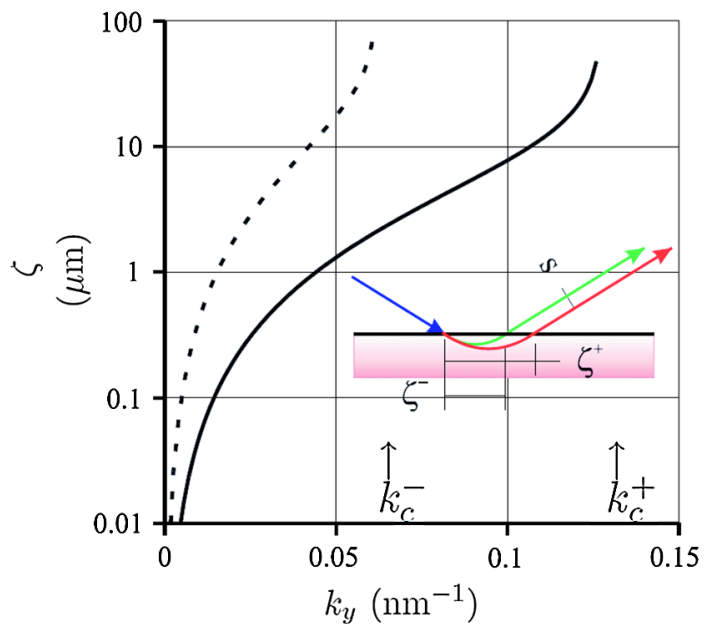

FIG. 2 (color online). Goos-Hänchen shift, $\zeta$ along the interface for an incident angle of $2 \mathrm{mrad}$ as function of the wave vector component perpendicular to the surface, $k_{y}$ for up (full line) and down (dashed line) spin state for fully magnetized iron. Inset: Splitting, $s$ of the neutron wave function at the interface. 
The transmission of the beam over this distance through the substrate material with an absorption (represented by the imaginary part of the potential, $-i \Sigma_{a} k \hbar^{2} / 2 m$, where $\Sigma_{a}$ is the macroscopic absorption cross section [16]) can be calculated by $\exp \left(-\zeta \Sigma_{a}\right)$. This is the same as the reduction of the reflectivity due to the imaginary part of the potential. This method was used by Pleshanov [9].

As was already noted by Mezei in 1980 [20], the polarization of a neutron beam can be interpreted as the degree of coherence between the wave functions representing the two spin states of neutron. Then, for a statistically stationary beam, the local polarization is defined as

$$
P_{j}(\vec{r})=\frac{\left\langle\left(\Psi^{+}(\vec{r}, t)^{*} \Psi^{-}(\vec{r}, t)^{*}\right) \hat{\sigma}_{j}\left(\begin{array}{l}
\Psi^{+}(\vec{r}, t) \\
\Psi^{-}(\vec{r}, t)
\end{array}\right)\right\rangle_{t}}{\left\langle\Psi^{+}(\vec{r}, t) \Psi^{+}(\vec{r}, t)^{*}\right\rangle_{t}+\left\langle\Psi^{-}(\vec{r}, t) \Psi^{-}(\vec{r}, t)^{*}\right\rangle_{t}},
$$

where the index $j$ denotes the $x, y$, or $z$ component, $\langle x(t)\rangle_{t}$ denotes the time average of $x(t)$, and $X^{*}$ the complex conjugated value of the function $X . \rho^{ \pm}\left(k_{y}\right)$ can be rewritten as $e^{i\left[\gamma\left(k_{y}\right) / 2\right]} e^{\left\{ \pm i\left[\delta\left(k_{y}\right) / 2\right]\right\}}$ where $\gamma\left(k_{y}\right)=\phi^{+}\left(k_{y}\right)+\phi^{-}\left(k_{y}\right)$ and $\delta\left(k_{y}\right)=\phi^{+}\left(k_{y}\right)-\phi^{-}\left(k_{y}\right)$. Using the above definition of the polarization vector, the matrix can be interpreted as a Larmor precession over an angle $\delta\left(k_{y}\right)$ of the polarization vector, the so-called Larmor pseudo precession [10]. Hence, the difference in phase shift between the up- and down-spin state can be determined by measuring the polarization change of the incident beam after total reflection at the sample surface. So, the only specific needs to measure the effect with neutrons is a neutron reflectometer with a possibility to measure the polarization change. OffSpec [11] is able to accurately measure this polarization change. Under the condition that the spin echo of the experiment does not change while changing the sample for a reference sample, the ratio of the measured spin-echo signal $(P)$ with a sample and with a reference $\left(P_{0}\right)$ is simply given by

$$
\frac{P}{P_{0}}=\cos N \delta\left(k_{y}\right)
$$

where $N$ is the number of reflections (at each reflection the shift is the same).

The most obvious sample to use would be a flat polished iron surface. However, a relatively strong magnetic field is needed to fully magnetize iron which would make it more difficult to have a well-defined spin echo. Therefore, a layer of $3 \mu \mathrm{m}$ Permalloy $\left(\mathrm{Fe}_{0.2} \mathrm{Ni}_{0.8}\right)$ was deposited onto a silicon wafer strip with dimensions of $20 \times 100 \times$ $0.8 \mathrm{~mm}^{3}$. The Permalloy layer is thin and fully magnetized in the guide field of the neutrons; therefore, the magnetic flux is parallel to the $z$ direction. However, small deviations well below 0.1 rad from this direction are of no consequence as they influence the polarization as a cosine effect. Any sample inhomogeneity would introduce depolarization effects, which have not been observed. A quartz plate (3 $\mathrm{mm}$ thickness) was used as a reference.

The ratio of the measured polarization (sample divided by reference) as a function of wave vector is shown in

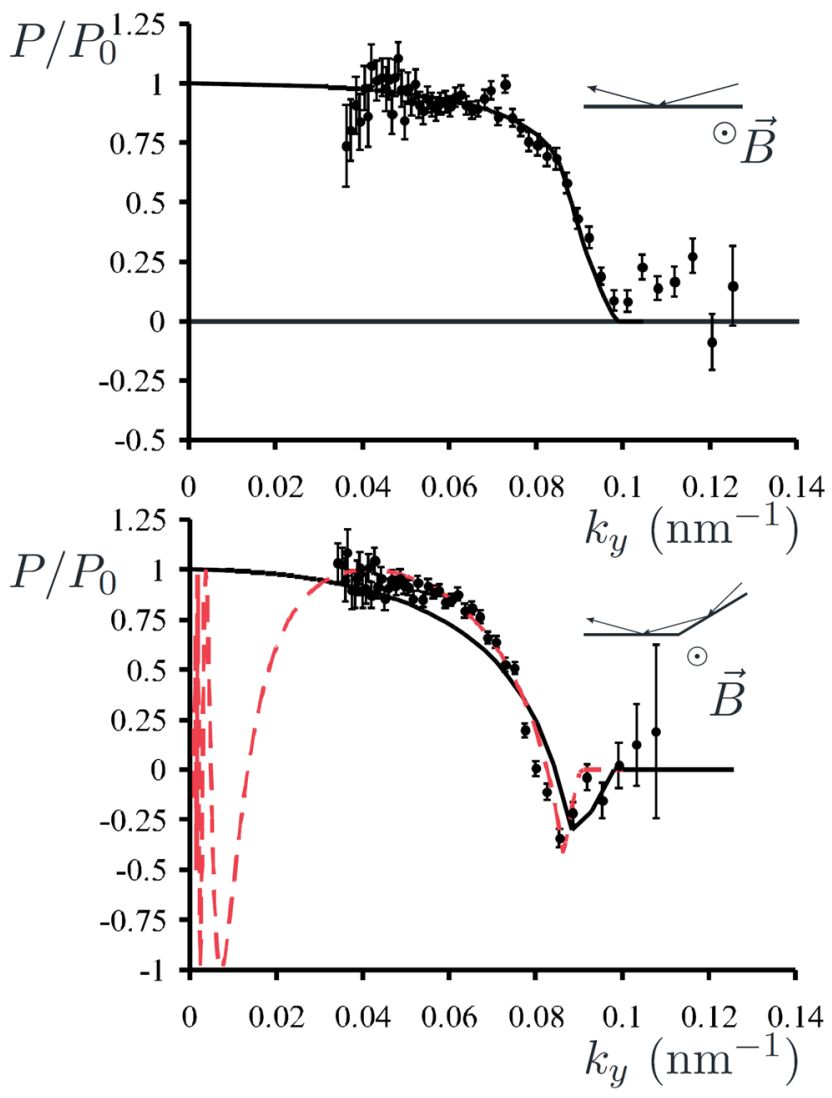

FIG. 3 (color online). Measured normalized polarization, $P / P_{0}$ as function of perpendicular wave vector, $k_{y}$ representing the Larmor pseudo precession due to the Goos-Hänchen shift along the interface for a single (top) and double (bottom) reflection from a Permalloy thin film. The black lines represent the theoretical predictions. The (red) dashed line in the lower graph represents a simulation (see text).

Fig. 3. The upper panel corresponds to a single reflection and the lower panel to a double reflection. The theoretical values were obtained by using literature values for the nuclear optical potential and saturation magnetization $(1 \mathrm{~T})$; hence, $k_{c}^{+}=0.120 \mathrm{~nm}^{-1}$ and $k_{c}^{-}=0.092 \mathrm{~nm}^{-1}$. The single reflection was obtained with an incident angle, $\theta$ of $5.0 \mathrm{mrad}$ and divergence (FWHM) of $0.35 \mathrm{mrad}$ (due to the waviness of the sample and the resolution of the detector used). As can be inferred from Fig. 2, the shift of the down spin is much larger than the shift of the up one; hence, from the definition of $\delta$ and Eq. (3), it can be shown that $\zeta^{-} \approx 2[1 / \sin (\delta / 2)-\sin (\delta / 2)] /\left(k_{c}^{-} \sin \theta\right)$, which yields for $k y=0.06 \mathrm{~nm}, \zeta^{-}=2.4 \mu \mathrm{m}$ and for $k y=$ $0.09 \mathrm{~nm}, \zeta^{-}=20 \mu \mathrm{m}$. For $\zeta^{+}$, these values are 1.0 and $2.8 \mu \mathrm{m}$, respectively. The double reflection was obtained by reflection in series, where the first reflection angle was $4.05 \mathrm{mrad}$ and the second angle $3.73 \mathrm{mrad}$. The angular divergence (FWHM) of $0.17 \mathrm{mrad}$ is smaller than before; as for the double reflection, the samples were rotated by 90 degrees, having a much smaller size in the beam direction. This reduces the influence of the waviness of the sample on the angular divergence of the beam. The single 
reflection data agree well with the theoretical predictions within the measurement accuracy. The double reflection data show, by becoming less than zero, that the shift does result in a rotation of the neutron polarization and is not a loss of spin-echo signal. The theoretical calculations predict the correct amount of polarization rotation (the minimum for the measurement and theory is the same), but there is deviation from the measured data that can be attributed to the more complex double reflection geometry (two slightly different angles) and a small magnetic field density anisotropy in the sample (the sample was rotated 90 degrees). Further, it is possible that the spin echo for the empty beam is not perfect, resulting in a wavelength dependent shift. This shift should be added to the shift in Eq. (5). If a simulation is performed taking these effects into account, the (red) dashed line is obtained, agreeing with the measured data. In the simulation, the measured angle and a block-shaped angular-resolution function were used. The magnetic field density was increased to $1.2 \mathrm{~T}$, not unusual for this type of material. The wavelength dependent shift was taken as $-1 \mathrm{rad} / \mathrm{nm}$. This introduces the oscillations at small $k_{y}$ (large wavelength).

The Goos-Hänchen shifts of the up- and down-spin states along the surface of the sample are different (see also inset in Fig. 2). Hence, after reflection, the up- and down-spin states are split in space. The amount of splitting, $s$ is equal to the difference in the Goos-Hänchen shift multiplied by the incident angle. For a single reflection, this splitting can increase up to $s=100 \mathrm{~nm}$. The transverse coherence length of the neutron beam is determined by the instrument configuration. For instance, the transverse coherence length reduces with increased diaphragm widths. Hence, by adjusting the instrumental configuration, the transverse coherence length of the neutron beam can be made less than the beam splitting induced by the GoosHänchen shift. Then, the resulting beam polarization will be less than the predicted value according to Eq. (5). When the diaphragm settings are reduced, the transverse coherence length increases and the resulting beam polarization reaches Eq. (5) again. However, when the interference between the up- and down-spin state is only determined by the intrinsic properties of the neutron, such measurements could provide a lower limit for the coherence length of the neutron as a wave packet.

For photons, the Goos-Hänchen shifts are accompanied by a delay time [21]. For neutrons, this delay time can be estimated by dividing the Goos-Hänchen shift by the neutron velocity [22] which gives

$$
\tau^{ \pm}=\frac{m}{\hbar\left(k_{c}^{ \pm}\right)^{2}} \frac{2 k_{y}}{\sqrt{\left(k_{c}^{ \pm}\right)^{2}-k_{y}^{2}}} .
$$

For the sample considered here, this is of the order of $0.1 \mu$ s close to the critical edge. This value can be greatly enhanced as, according to the above formula, it is proportional to the inverse of the scattering potential. Hence, by reducing the scattering potential, the delay time can be increased. This can be understood as the neutrons taking more time to bounce back from a surface when the forces on the neutron are less strong. The OffSpec instrument is able to measure both spatial splitting and time delay by adjusting the instrumental configuration. This creates the possibility to check if the delay time can be inferred from the shift according to the above assumptions.

In conclusion, the difference in the Goos-Hänchen shifts for spin-polarized neutrons during reflection has been experimentally observed and is well described within a simple theoretical framework. The results suggest that the accompanying shift in time should also be observable. Moreover, the observed spatial shift can be used to accurately determine the coherence properties of the neutron beam. The experiment sets the basis for new extended studies using neutron quantum mechanics for an increased range of topics.

[1] I. Newton, Opticks, or, A Treatise of the Reflections, Refractions, Inflections and Colours of Light (Prometheus Books, New York, 2003), 4th ed., Chap. Book Three, Part I, query 29 (London, 1730), pp. 370374.

[2] F. Goos and H. Hänchen, Ann. Phys. (Leipzig) 436, 333 (1947).

[3] F. Goos and H. Hänchen, Ann. Phys. (Leipzig) 440, 251 (1949).

[4] C. W. J. Beenakker, R. A. Sepkhanov, A. R. Akhmerov, and J. Tworzydo, Phys. Rev. Lett. 102, 146804 (2009).

[5] M. Merano, A. Aiello, M. P. van Exter, and J.P. Woerdman, Nat. Photon. 3, 337 (2009).

[6] F. Pillon, H. Gilles, and S. Girard, Appl. Opt. 43, 1863 (2004).

[7] M. Maaza and B. Pardo, Opt. Commun. 142, 84 (1997).

[8] V. Ignatovich, Phys. Lett. A 322, 36 (2004).

[9] N. Pleshanov, Physica B (Amsterdam) 198, 70 (1994).

[10] B. Toperverg, H. Lauter, and V. Lauter-Pasyuk, Physica B (Amsterdam) 356, 1 (2005).

[11] R. M. Dalgliesh and S. Langridge, ISIS Second Target Station (2009).

[12] J. Plomp, V. O. de Haan, R. M. Dalgliesh, S. Langridge, and A. A. van Well, Thin Solid Films 515, 5732 (2007).

[13] B. Rohwedder, Phys. Rev. A 65, 043619 (2002).

[14] F. Pillon, H. Gilles, S. Girard, M. Laroche, R. Kaiser, and A. Gazibegovic, J. Opt. Soc. Am. B 22, 1290 (2005).

[15] P. A. M. Dirac, Rev. Mod. Phys. A 117, 610 (1928).

[16] V.F. Sears, Neutron Optics (Oxford University Press, Oxford, 1989), 1st ed..

[17] N. Pleshanov, Zeitschrift für Physik B 100, 423 (1996).

[18] R. Renard, J. Opt. Soc. Am. 54, 1190 (1964).

[19] H. Lotsch, J. Opt. Soc. Am. 58, 551 (1968).

[20] F. Mezei, in Imaging Processes and Coherence in Physics, Lecture Notes in Physics Vol. 112, edited by M. Schlenker (Springer-Verlag, Berlin, 1980), p. 282.

[21] D. Chauvat, C. Bonnet, K. Dunseath, O. Emile, and A. L. Floch, Phys. Lett. A 336, 271 (2005).

[22] H. Friedrich, A. Jurisch, and E. Mesfin, J. Phys. Conf. Ser. 99, 012008 (2008). 